\title{
Post-radiation soft tissue damage in endometrial carcinoma: a case report
}

\author{
Strahil Asenov Strashilov ${ }^{1}$, Vasil Nanev¹, Stanislav Slavchev², Denislava Ivanova ${ }^{3}$, Stoyan Kostov², \\ Angel Yordanov ${ }^{1}$
}

${ }^{1}$ Medical University of Pleven, Bulgaria

${ }^{2}$ Medical University Varna, Bulgaria

${ }^{3}$ University Hospital Sofiamed, Bulgaria

\begin{abstract}
Introduction: Radiotherapy (RT) is a widely used treatment modality of malignant tumours of the uterine cervix and body. There are different techniques to deliver RT to the tumour lesions, including external beam radiation and brachytherapy. All international guidelines recommend RT as treatment modality for many stages of uterine cervical and body cancers because it improves a number of oncological outcomes, such as diseasefree and overall survival. However, it may also lead to a number of complications, which can be roughly divided into early or late, depending on the time of their manifestation. The most frequent RT-induced early complications involve the skin and the soft subcutaneous tissues. They typically present as inflammatory conditions of all abdominal wall layers: dermatitis, cellulitis, and necroses of subcutaneous fatty tissue, muscles, or fasciae.

Case report: This paper presents a case report of a 38-year-old woman diagnosed with endometrial cancer (EC). She was initially treated with open abdominal surgery, and subsequently the treatment was continued with external beam adjuvant RT.

Discussion: While RT was ongoing, a necrosis of the anterior abdominal wall in the surgical scar developed. It manifested at the end of the RT treatment and is thus regarded as an early complication of the RT for EC. It was successfully managed with surgery, and there was no compromise in the treatment of the oncological condition.

Conclusions: RT-induced necrosis of the surgical scar of the anterior abdominal wall is a very rare complication. Surgical treatment is the main method of treatment of this condition.
\end{abstract}

Key words: radiation therapy, radiation-induced complications, endometrial cancer.

\section{Introduction}

Radiation therapy or radiotherapy (RT) is essential in the treatment of cancers of the uterine cervix or body. It has evolved significantly during the last decades and currently includes a number of methods for external beam radiation or brachytherapy with different planning and delivery systems. RT leads to a dramatic change in the evolution of oncological conditions, such as cervical cancer (CC) or endometrial cancer (EC), and improved survival of patients with these cancers. However, RT may also induce a number of complications, which may be directly related to the anatomy of the lesser pelvis, the localization and the spread of the EC or CC, or aggravated by previous RT, or systemic or surgical treatments in the area. Toxicity of RT depends on the total radiation dose, treatment volume, and normal tissues in the vicinity. Additionally, different tissues take different times to develop sequelae from RT. Regarding the onset of RT-induced complications, they may be divided into early or acute (developing during or within the first 4 weeks after radiation); subacute (developing at 1-3 months) and late ( $>90$ days after initiation of RT) [1]. The most frequent complications of RT are related to damage of the skin and the soft tissues within the radiation field. These are dermatitis, cellulitis, as well as necroses of the subcutaneous adipose tissue, muscles, and fasciae [2].

\section{Case report}

A 38-year-old woman with no comorbidities was diagnosed with EC due to complaints of vaginal discharge between periods. The disease was histologically confirmed via abrasion, and after imaging and clinical examination the disease was clinically staged as per TNM 8 as pT3ap NO MO. Open surgery (laparotomy) was the initial treatment approach: Pfannenstiel skin incision, followed by layer-by-layer dissection of the abdominal wall and gradual approach to the lesser pelvis. Total hysterectomy and bilateral adnexectomy 


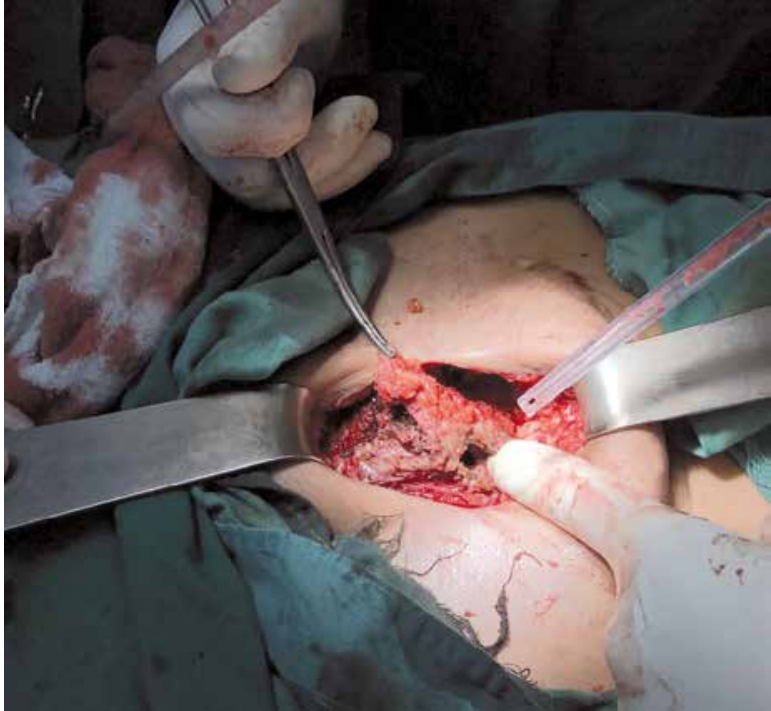

Fig. 1. Intraoperative findings

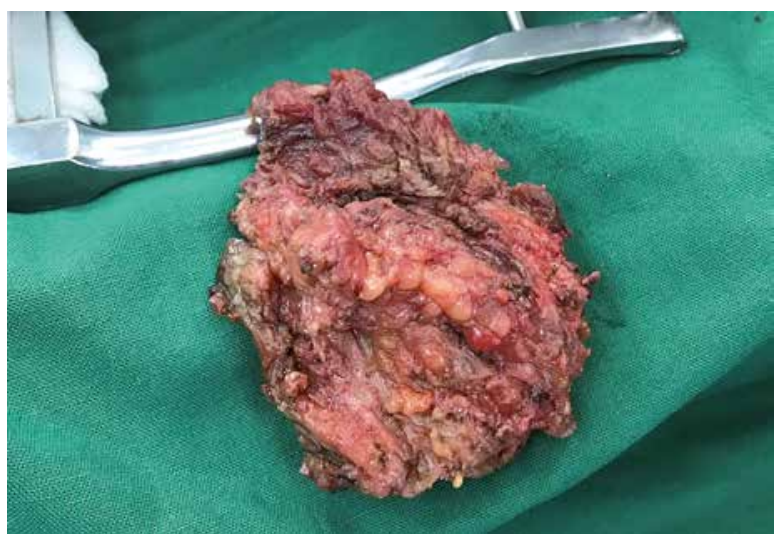

Fig. 2. Macroscopic appearance of the preparation

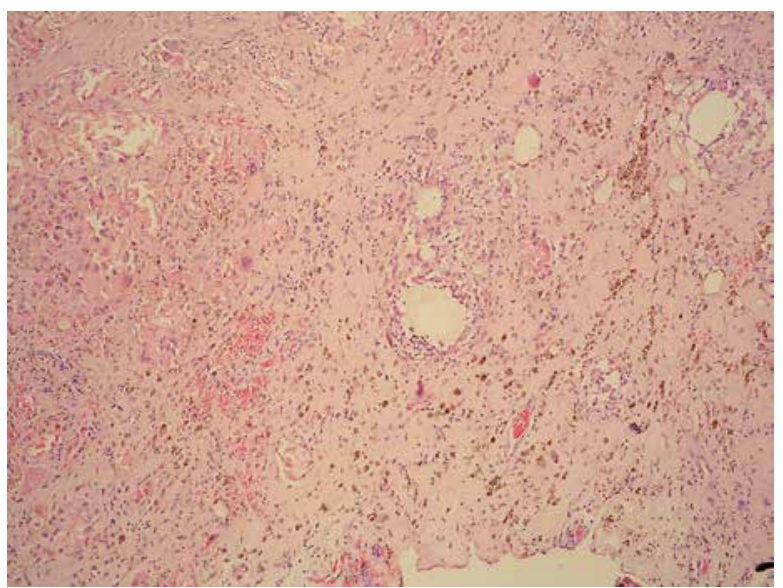

Fig. 3. Histopathological image of the removed formation

was done in view of the above-described EC. After RO tumour resection the abdomen was closed: the peritoneum was closed via a long suture with absorbable surgical threads; the incision of the muscles of the anterior abdominal wall was closed with single stitches using non-absorbable silk surgical threads. The subsequent closure of the abdominal cavity was again done as a typical layer-by-layer suture, and the subcutaneous connective tissues were closed with single stitches, using absorbable surgical threads. The skin defect was closed with single stitches via non-absorbable monofilament surgical threads.

The final histology report confirmed endometroid EC without myometrial invasion and with no lymph node involvement; a metastasis to the left ovary was identified, and thus the postoperative TNM-8 disease stage was pT3A pNO MO. The stage of the disease indicated the need of adjuvant treatment, and external beam RT was prescribed. After primary healing of the surgical incision RT was initiated 4 weeks after surgery. The volumetric-modulated arc therapy (V-MAT) delivered RT via a linear accelerator, using a cone beam, continuously rotating around the patient. A 2-Gy daily dose and a total dose of 56 Gy were delivered to the tumour bed in the lesser pelvis.

One month after the last day of RT, the patient reported having pains above the symphysis in the area of the Pfannenstiel incision scar. She did not report any other symptoms such as fever or chills. A computed tomography of the abdomen and lesser pelvis identified a rounded lesion, considered as "an abscess" in the lower anterior abdominal wall, involving the fascia of the abdominal rectal muscle. Gynaecological examination identified a post-hysterectomy normal vagina with no sign of recurrence. All laboratory tests, including peripheral blood count, and biochemical and urine tests, were within the normal ranges. Surgical management of the local inflammatory process was the initial step. In the tissues of the anterior abdominal wall a solid lesion, involving hypoderm, fascia, and muscles, was found (Fig. 1). The mass was entirely preperitoneal and was resected with clear resection margins (Fig. 2), and the defect of the fascia and abdominal wall muscles was closed using a mesh.

The final histology result with Van Gieson stains identified fatty fibrous tissue material and striated muscles; presence of lipogranulomas, foreign body granulomas, and necrosis, surrounding a pseudocyst wall in incomplete transition to connective tissue. There was no cancer tissue, and only chronic inflammatory infiltrate with pigmented macrophages and hyalinosis were found (Fig. 3).

The surgical incision healed primarily and until now, at 6 months of follow-up, the patient has not reported any further complaints or complications. She is also free of the oncological disease (EC) at present.

\section{Discussion}

Radiotherapy to tumour lesions in the lesser pelvis may induce toxicity to the gastrointestinal or genitouri- 
nary tract, haematologic toxicity, as well as radiation-induced changes to the bones, skin, and subcutaneous tissues. RT-induced complications may be subclassified, according to the time of development, as acute, subacute, and late. Acute toxicity develops within days to 4 weeks during or after radiation and is frequently entirely reversible. In contrast, late toxicity that develops more than 3 months after RT is frequently severe and irreversible [1].

Early RT-induced injury of the skin and the subcutaneous soft tissues, especially those in the surgical incision area, is observed in $10-50 \%$ of cases with cervical and endometrial carcinoma [3, 4] and in $85-100 \%$ of vulvar carcinoma cases [5-7]. RT-induced early damage, along with its onset, severity, and duration, may directly affect the clinical evolution of the oncological condition and may introduce a delay or cancellation of a potentially curative treatment such as RT or subsequent systemic treatment. Vascular diseases, imbalanced nutrition, and smoking may additionally impair the longterm recovery of the skin and the subcutaneous soft tissues, thus resulting in post-radiation complications [5]. Early injury of the subcutaneous soft tissues and the surgery cicatrix that fall within the RT area is due to vascularization problems and a suppressed or delayed recovery process [2].

In our case, the lesion in the abdominal wall was in fact an inflammatory necrotic mass in the scar area from the surgery for EC to the lesser pelvis. The skin integrity was interrupted due to the skin incision for the laparotomy, but it has healed primarily and completely prior to initiation of RT. We suppose a cause-effect relationship that clearly suggests that the necrotic fibrotic lesion formed as a consequence of the percutaneous RT. As a new event, which occurred about a month after the start of RT, it is considered to be an early RT-related complication because it formed and evolved while the patient was still on RT.

We looked in the published literature to find similar reports, but to the best of our knowledge this is the first described case. A necrosis of the abdominal wall within the surgical cicatrix is a very rare complication, but as an early complication it may compromise a potentially curative treatment such as adjuvant RT. It is important not to delay the diagnosis and to recognize the condition in order to organise its timely management. The imaging followed by surgical resection resulted in complete recovery of the complication.

\section{Conclusions}

A necrotic tumour mass in the skin scar after previous surgical treatment for an oncological condition may be a diagnostic challenge because it needs to be differentiated between a benign inflammatory condition and potential tumour involvement. Either of these conditions needs timely management and treatment. Despite the fact that clinical examination and proper imaging are essential, the final diagnosis is confirmed only after surgery, which is the main treatment modality. It is impossible to predict or prevent such complications, but raising awareness among gynaecological oncologists might facilitate its diagnosis and management. Because RT is a treatment aimed at curing potentially lethal oncological conditions such as cervical or EC, early identification and timely management of its complications is important. We hope that this case report may help to avoid mistakes or delays in the diagnosis of such an RT-induced early complication.

\section{Disclosure}

The authors report no conflict of interest.

\section{References}

1. Herrmann T, Knorr A, Dörner K. The RTOG/EORTC classification criteria for early and late radiation reactions. Radiobiol Radiother (Berl) 1987; 28: 519-528.

2. Viswanathan A, Lee L, Eswara J, et al. Complications of pelvic radiation in patients treated for gynecologic malignancies. Cancer 2014; 120: 38703883.

3. Keys H, Roberts J, Brunetto V, et al. A phase III trial of surgery with or without adjunctive external pelvic radiation therapy in intermediate risk endometrial adenocarcinoma: a Gynaecologic Oncology Group study. Gynecol Oncol 2004; 92: 744-751.

4. Rose P, Bundy B, Watkins E, et al. Concurrent cisplatin-based radiotherapy and chemotherapy for locally advanced cervical cancer. N Engl J Med 1999; 340: 1144-1153.

5. Beriwal S, Shukla G, Shinde A, et al. Preoperative intensity modulated radiation therapy and chemotherapy for locally advanced vulvar carcinoma: analysis of pattern of relapse. Int J Radiat Oncol Biol Phys 2013; 85: $1269-1274$.

6. Moore D, Thomas G, Montana G, et al. Preoperative chemoradiation for advanced vulvar cancer: a phase II study of the Gynaecologic Oncology Group. Int J Radiat Oncol Biol Phys 1998; 42: 79-85.

7. Mak R, Halasz L, Tanaka C, et al. Outcomes after radiation therapy with concurrent weekly platinum-based chemotherapy or every-3-4-week 5-fluorouracil-containing regimens for squamous cell carcinoma of the vulva. Gynecol Oncol 2011; 120: 101-107. 